\title{
A New Equalizer Structure for High-Speed Optical Links based on Carrierless Amplitude and Phase Modulation
}

\author{
Nikos Bamiedakis, Xiaohe Dong, David G. Cunningham, Richard V. Penty and Ian H. White \\ Electrical Engineering Division, Department of Engineering, University of Cambridge, \\ 9 JJ Thomson Av., CB3 OFA, UK \\ e-mail:nb301@cam.ac.uk
}

\begin{abstract}
Spectral efficient modulation formats can enable the transmission of higher data rates than conventional on-off keying (OOK). Carrierless amplitude and phase modulation (CAP) is such an attractive modulation scheme that has been widely considered for use in different types of optical links. The scheme however can suffer from intersymbol interference (ISI) and channel crosstalk (CCI) when the frequency response of the channel is not ideal. Conventional equalizers based on feedforward (FFE) and decision feedback (DFE) equalizers are easy to implement in practice and can mitigate some of the induced ISI. However, they fail to suppress the induced CCI in the link as each channel is equalized independently. As a result, we have recently proposed the use of a new equalizer structure for use in CAP-based optical links to mitigate these transmission impairments. This new equalizer, named CAP equalizer, can be formed with conventional FFEs and DFEs with minimal additional complexity whilst providing significant performance advantages. In this paper therefore, we review the equalizer structure and report recent demonstrations of its use in short-reach optical links. We present experimental studies on a $112 \mathrm{~Gb} / \mathrm{s}$ CAP-16 VCSEL-based OM4 MMF link and a $4 \mathrm{~Gb} / \mathrm{s}$ CAP-16 LED-based POF link and compare the performance of the links when both a conventional FFE and DFE equalizer and the newly proposed CAP equalizer are used. The results clearly demonstrate that the CAP equalizer offers improved receiver sensitivity and enables successful data transmission over longer fibre reaches.
\end{abstract}

Keywords: carrierless amplitude and phase modulation, equalization, short-reach optical links, multimode fibre links, vertical-cavity surface emitting lasers, light emitting diodes, plastic optical fibre links

\section{INTRODUCTION}

The ever increasing data traffic and the advent of new applications are pushing the demand for high-speed short-reach optical links both in data centres and end-user environments such as in in-home networks [1]. Examples of such links include vertical-cavity surface-emitting laser-based (VCSEL-based) multimode fibre (MMF) links that comprise the vast majority of short-reach links inside data centres and light emitting diodebased (LED-based) free-space and plastic optical fibre (POF) links that are increasingly used inside homes and cars. However, the high-speed performance of these low cost optical links is typically limited by the low bandwidth of the optical and electrical components used. As a result, spectrally-efficient modulation schemes such pulse amplitude modulation (PAM), discrete multitone modulation (DMT) and carrierless amplitude and phase modulation (CAP), are increasingly considered for use in such optical links as they can enable the transmission of higher data rates than conventional on-off keying (OOK). In recent years, multiple demonstrations of their use in these different types of short-reach optical links have been reported: VCSELbased MMF links [2-5], LED-based free space [6-9] and POF links [10-15].

CAP modulation in particular, is an attractive passband modulation scheme that offers increased spectral efficiency over OOK, removes baseline wander and allows simple implementation that can be achieved with digital circuits[16]. It relies on the transmission of two orthogonal pulses which form a Hilbert transform pair and which can be perfectly separated at the received side provided that the magnitude of the frequency response of the link is flat and its phase response is linear. In real systems however, this is not the case resulting in intersymbol interference (ISI) and channel crosstalk interference (CCI) which severely impairs the performance of the link [17]. We have recently proposed a new equalizer structure that mitigates such transmission impairments in CAP-based links and which can easily be implemented using hardware [17]. In this article therefore, we review the principle of operation of this new equalizer, named CAP equalizer, and present experimental studies that demonstrate the performance improvements it can bring in short-reach optical links. The structure of this novel equalizer is similar to that of conventional feedforward (FFE) and decision feedback (DFE) equalizers and therefore, can be implemented with minimal additional complexity. The performance of a VCSEL-based CAP-16 OM4 MMF link operating at $112 \mathrm{~Gb} / \mathrm{s}$ [17] and a LED-based POF link operating at 4 $\mathrm{Gb} / \mathrm{s}[18]$ are assessed when both the newly proposed CAP equalizer and the conventional FFE and DFE equalizer is employed. The total number of taps of both equalizers is kept the same in order to conduct a fair comparison of the obtained link performance. It is demonstrated that the use of the CAP equalizer provides significant improvements in receiver sensitivity and enables the transmission of the data signals over longer reach in comparison to the conventional FFE and DFE equalizer. 
The rest of the paper is structured as follows. Section 2 presents the principle of operation and basic structure of the new equalizer structure. Section 3 reports the experimental data transmission studies on the use of this equalizer in a short-reach VCSEL-based OM4 MMF link and a LED-based POF link respectively. Finally, Section 4 provides the conclusions.

\section{THE CAP EQUALIZER}

Figure 1 shows the typical transmission system based on CAP modulation of order $M$ and employing conventional FFE and DFE equalizers. At the transmitter, the input data is divided in groups of $\log _{2} M$ bits which are appropriately encoded in multi-level complex symbols $S_{i}$ using standard quadrature amplitude modulation (QAM) mapping and up-sampled. The real part of the generated symbols modulates the in-phase (I-channel) pulse shaping filter $p_{I}(t)$ while the imaginary part the quadrature (Q-channel) one $p_{Q}(t)$. The two orthogonal signals are superimposed appropriately and are used to modulate the optical transmitter in the link. After transmission over the optical link and the optical to electrical conversion by the optical receiver, the received signal is passed through the matched filter of the I- and Q-channel with impulse response $p_{M . I}(t)$ and $p_{M . Q}(t)$ respectively. The response of the matched filters consists of the time reversed version of the pulse shaping function used at the transmitter: $p_{M . I}(t)=p_{I}(-t)$ and $p_{M . Q}(t)=p_{Q}(-t)$. The orthogonality of the pulse shaping functions ensures that the two channels can be perfectly separated at the receiver provided that the channel has a frequency response with flat amplitude and linear phase over the bandwidth of the transmitted signals [17]. In the real world however, this condition is typically not true, destroying therefore the orthogonality of the two channels and resulting in intersymbol interference (ISI) and channel crosstalk interference (CCI) in the link. As a result, equalization is applied at the receiver to mitigate these impairments and recover the transmitted symbols. The recovered symbols are then down-sampled, decoded and combined appropriately to generate the transmitted input data.

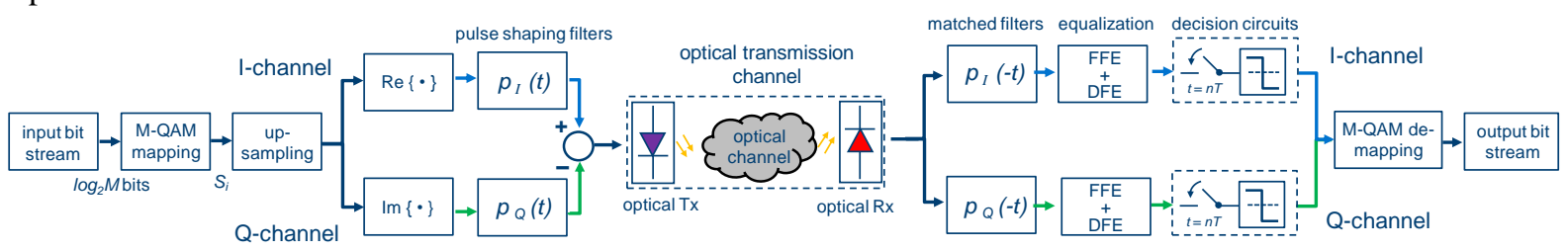

Figure 1. Typical transmission system based on CAP modulation and conventional FFE and DFE equalizers.

Typically, the equalization applied is based on conventional FFEs and DFEs as these are relatively simple to implement in hardware. More complex equalizers, such as non-linear Volterra equalizers [19-22] and those based on machine-learning algorithms [23-25], have been also proposed for use in CAP-based transmission systems and have been demonstrated in optical links. However, these are more complex to implement or require significant digital signal processing. Conventional FFE and DFE equalizers can suppress the ISI in the link, but fail to efficiently mitigate the CCI as they rely only on the information transmitted on each channel (Figure 2) [17]. In order to overcome this issue, we have recently proposed the use a new equalizer structure, named the CAP equalizer, which is based on conventional FFE and DFEs but utilises the signal received on both channels to recover the symbols transmitted on each of them. Its principle of operation is illustrated in Figure 3(a) and its basic structure is shown in Figure 3(b). It is formed with conventional FFE and DFEs and therefore its practical implementation requires minimal additional complexity over the conventional scheme shown in Figure 2(b). The mathematical formulation of its operation has been presented in [17].

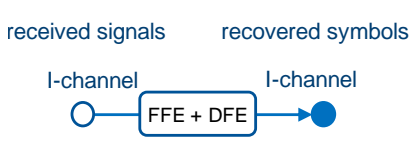

(a)

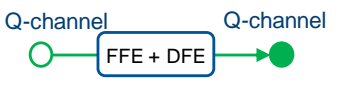

(b)

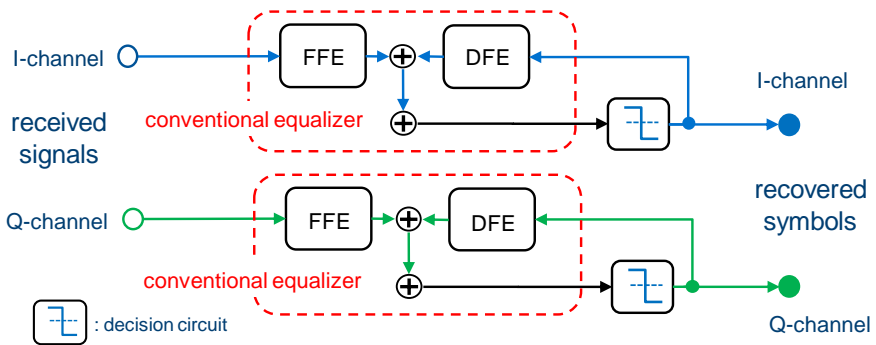

Figure 2. (a) Principle of operation and (b) basic structure of the conventional FFE and FFE equalizer. 


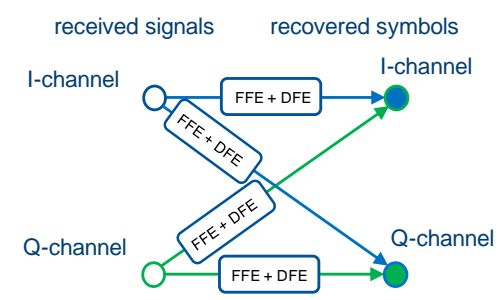

(a)

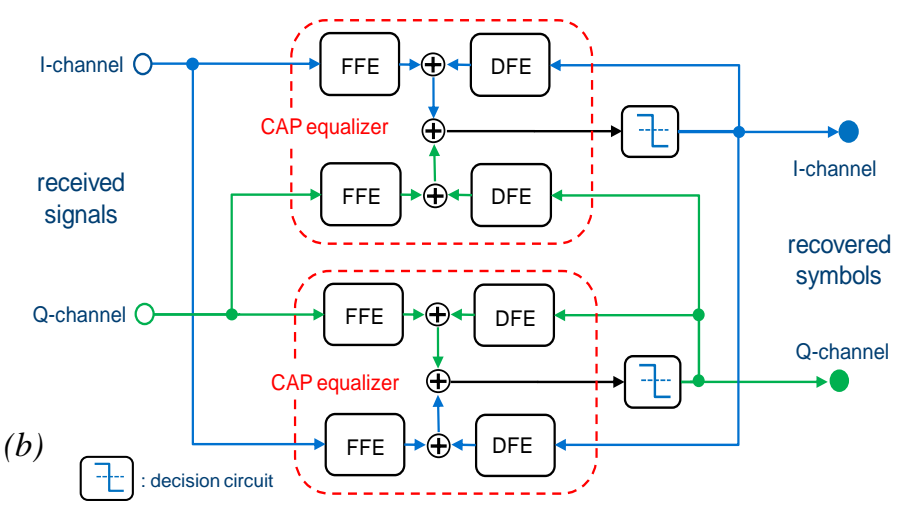

Figure 3. (a) Principle of operation and (b) basic structure of the recently proposed CAP equalizer.

\section{EXPERIMENTAL STUDIES}

The use of the CAP equalizer is demonstrated in two types of short-reach optical links employing CAP-16 modulation: (i) a VCSEL-based OM4 MMF link operating at $112 \mathrm{~Gb} / \mathrm{s}$ [17] and (ii) a LED-based POF link operating at $4 \mathrm{~Gb} / \mathrm{s}$ [18]. The performance of each link is evaluated when both the CAP equalizer and a conventional FFE and DFE equalizer are employed. To allow fair comparison between the two configurations, the tap spacing (half the symbol period, T/2-spaced) and the total number of taps is kept the same for both equalizers while the same received signal is used as input for each equalizer. For both types of links, the CAP-16 modulating signal is generated with an arbitrary waveform generator (AWG) while the signal demodulation and equalization and BER estimation are carried out offline using Matlab. The equalizer taps are optimised based on the transmission of a training pattern and are calculated using the least mean squares (LMS) algorithm. The BER performance of the link is estimated after equalization is applied on a test pattern using the tap coefficients obtained via the training process. The noise performance used for the BER calculations is based on noise measurements carried out on the link for the same average received optical power, and the noise enhancement introduced by each equalizer. For the data transmission experiments described below, both the training and test pattern used are based on a $2^{11}-1$ pseudorandom binary sequence (PRBS). For the test pattern, the input bits are divided in groups of 4 bits and encoded in CAP-16 multi-level symbols. A similar process is carried out for the training pattern, but the 4-bit groups are randomly re-ordered to generate a different CAP-16 modulating signal.

\section{1 $112 \mathrm{~Gb} / \mathrm{s}$ CAP-16 VCSEL-based OM4 MMF link}

The experimental setup used is shown in Figure 4. An $850 \mathrm{~nm}$ multimode VCSEL with a 3 dB bandwidth of $\sim 25$ $\mathrm{GHz}$ is employed as the light source and is biased at $12 \mathrm{~mA}$ bias current. The modulating electrical signal is amplified to a $1.5 \mathrm{~V}$ peak-to-peak amplitude and is fed to the laser via a high-bandwidth bias tee. The emitted light is coupled into a short MMF patchcord using a pair of microscope objectives (16x) and is transmitted over the OM4 MMF. Two different OM4 fibre lengths are employed to test the performance of the link: $100 \mathrm{~m}$ and $150 \mathrm{~m}$. The back-to-back (b2b) link (no OM4) is also tested to obtain a reference for the link performance. A variable optical attenuator (VOA, Agilent NA7766A) is used to adjust the received optical power level. At the receiver end, a PIN photodiode with $30 \mathrm{GHz}$ bandwidth (D30-850M) and a $40 \mathrm{GHz}$ RF amplifier (SHF 807) are used to convert the received optical signal to their electrical counterpart and amplify it to appropriate amplitude for demodulation. The received electrical waveforms including at least 256 periods of the transmitted data pattern are captured using a digital phosphor oscilloscope (DPO, Tektronix DPO77002SX). The waveforms are averaged and offline processed. The waveforms are demodulated and equalized using both equalizers. The total number of taps of the two equalizers is the same and set to 27 taps for the FFE part and 27 taps for the DFE part. The CAP equalizer has 22 taps on the main channel and 5 taps on the crosstalk channel for each part. Figure 5 shows the obtained noise-free constellation diagrams for the different link lengths and equalizer applied. It can be clearly noticed that the CAP equalizer yields clear diagrams for all link lengths enabling successful recovery of the transmitted symbols. On the contrary, the conventional equalizer fails to recover the transmitted symbols for both the 100 and $150 \mathrm{~m}$ links. The BER plots for the links that achieve successful data transmission are shown in Figure 6. The receiver sensitivity to achieve the BER threshold of $3.8 \times 10^{-3}$ required for hard-decision forward error correction (HD FEC) is found to be $-3.8 \mathrm{dBm},-2 \mathrm{dBm}$ and $0.6 \mathrm{dBm}$ for the b2b, $100 \mathrm{~m}$ and $150 \mathrm{~m}$ OM4 MMF links respectively when the CAP equalizer is used. 


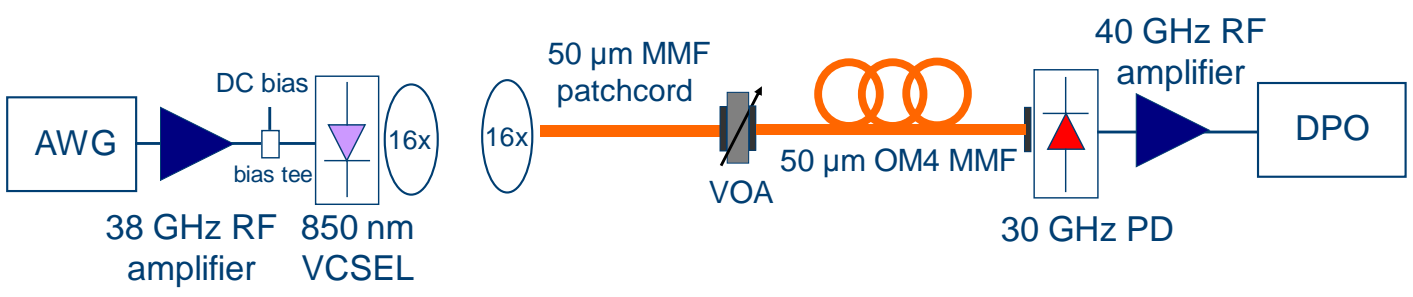

Figure 4. Experimental setup used in data transmission studies over the VCSEL-based OM4 MMF link.
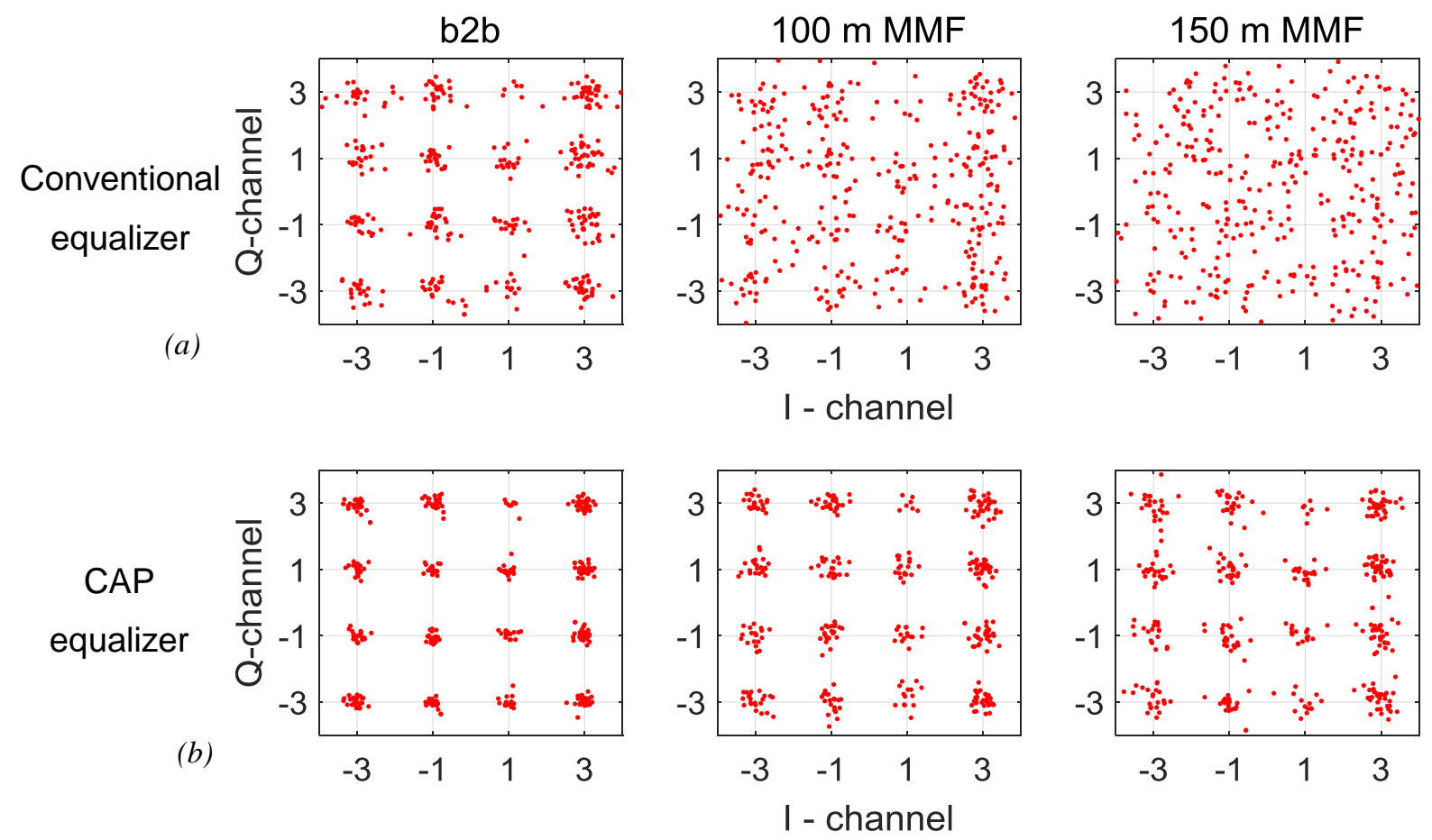

Figure 5. Constellation diagrams for $112 \mathrm{~Gb} / \mathrm{s} C A P-16$ transmission and $0 \mathrm{dBm}$ average received optical power for the different link lengths and the two equalizers used: (a) conventional equalizer and $(b)$ CAP equalizer.

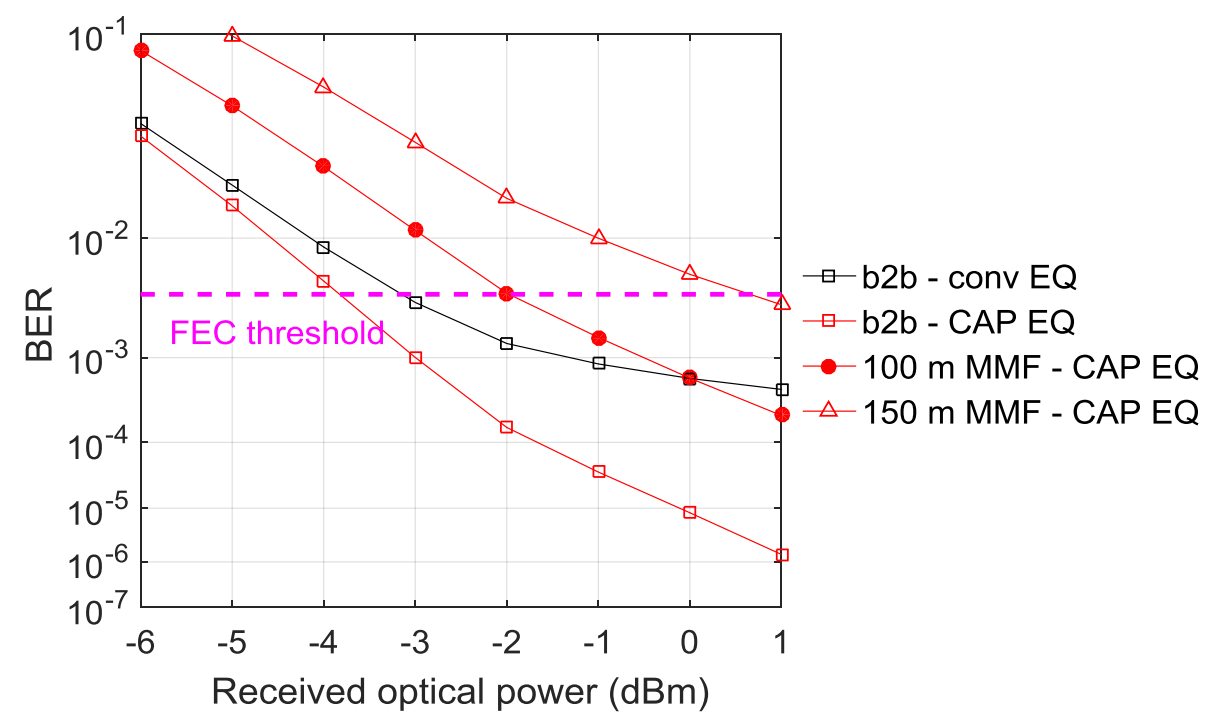

Figure 6. BER plots for the operating links for the different OM4 MMF lengths and the two equalizers. 


\subsection{Gb/s CAP-16 LED-based POF link}

The experimental setup used for the data transmission tests is shown in Figure 7. A micro-pixelated LED ( $\mu \mathrm{LED}$ ) with a $3 \mathrm{~dB}$ bandwidth of $\sim 135 \mathrm{MHz}$ at the bias current of $60 \mathrm{~mA}$ is used as the light source [26]. The POF used in these experiments consists of standard step-index POF with $1 \mathrm{~mm}$ core diameter (Eska Mega). Two different POF lengths are tested: 10 and $25 \mathrm{~m}$. The back-to-back (b2b) link which includes a short $(\sim 1 \mathrm{~m})$ POF patchcord is also tested for reference. The light emitted from the $\mu$ LED is coupled into the POF with an optical lens, while at the POF output a pair of optical lenses is used to focus the light onto an $800 \mu \mathrm{m}$ in diameter $\mathrm{Si}$ avalanche photodiode (APD, First Sensor AD800-11). A free-space VOA is used to adjust the level of the received optical power. The APD has a $3 \mathrm{~dB}$ bandwidth of $650 \mathrm{MHz}$. A $1 \mathrm{GHz}$ low noise amplifier (MiniCircuits ZFL-1000LN) is used to amplify the received signals. Multiple (> 256) periods of the transmitted waveforms are captured with the DPO and averaged. The obtained waveform is offline processed with Matlab which includes demodulation, equalization and BER estimation as described in the previous sections. Here, the total length of the two equalizers is 60 taps for the FFE part and 60 taps for the DFE one. The CAP equalizer uses in each part, FFE and DFE, 45 and 15 taps for the main and crosstalk channel respectively. Figure 8 shows the obtained constellation diagrams for the different POF lengths when both the conventional and CAP equalizer are employed. The CAP equalizer yields clear diagrams for all POF lengths under test and is able to recover the transmitted symbols. The conventional FFE and DFE equalizer of the same total tap length is able to recover the data transmitted for only the $\mathrm{b} 2 \mathrm{~b}$ and $10 \mathrm{~m}$ link. It fails to do so for the $25 \mathrm{~m}$ link. The respective BER curves for the operating links are shown in Figure 9. The links employing the CAP equalizer exhibit a receiver sensitivity for the FEC BER threshold of $1 \times 10^{-3}$ of $-18,-15.8$ and $-13 \mathrm{dBm}$ for the $\mathrm{b} 2 \mathrm{~b}, 10 \mathrm{~m}$ and $25 \mathrm{~m}$ POF respectively. The respective sensitivity values for the conventional equalizer are -17.6 and $-14.3 \mathrm{dBm}$ for the $\mathrm{b} 2 \mathrm{~b}$ and $10 \mathrm{~m}$ link respectively. The use of the CAP equalizer provides a significant improvement in receiver sensitivity over the conventional equalizer of $0.4 \mathrm{~dB}$ for the $\mathrm{b} 2 \mathrm{~b}$ link and $1.5 \mathrm{~dB}$ for the $10 \mathrm{~m}$ POF link.

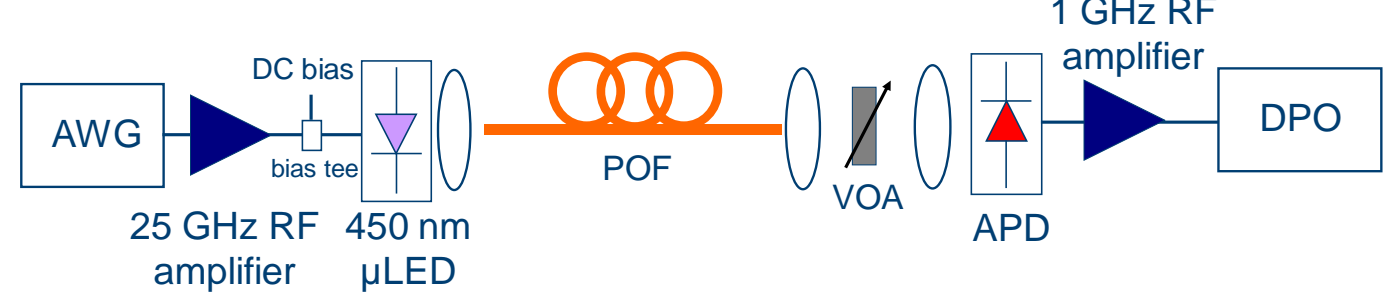

Figure 7. Experimental setup used in data transmission studies over the LED-based POF link.
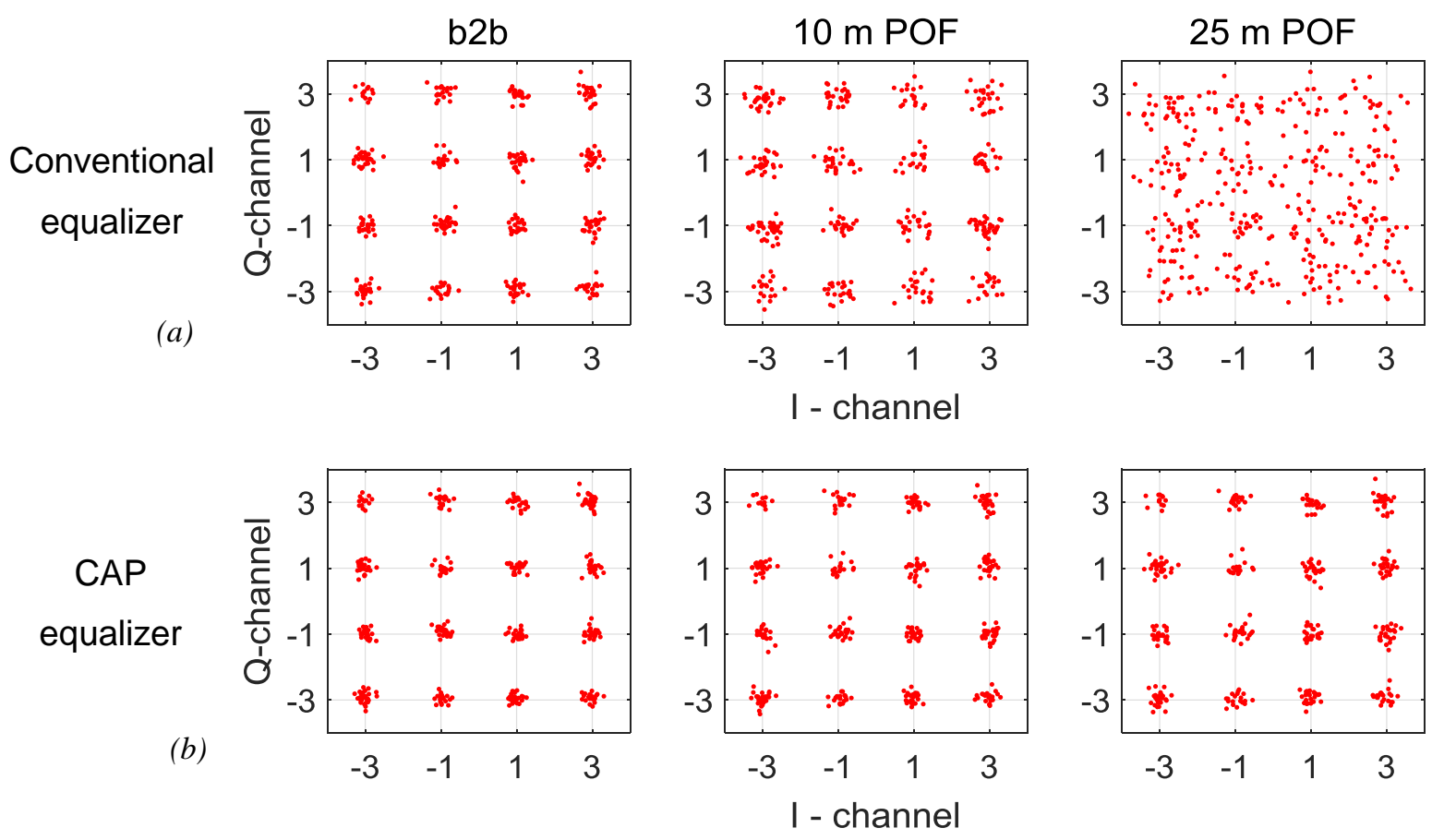

Figure 8. Constellation diagrams for $4 \mathrm{~Gb} / \mathrm{s}$ CAP-16 transmission and $-8.5 \mathrm{dBm}$ average received optical power for the different POF lengths and the two equalizers used: (a) conventional equalizer and (b) CAP equalizer. 


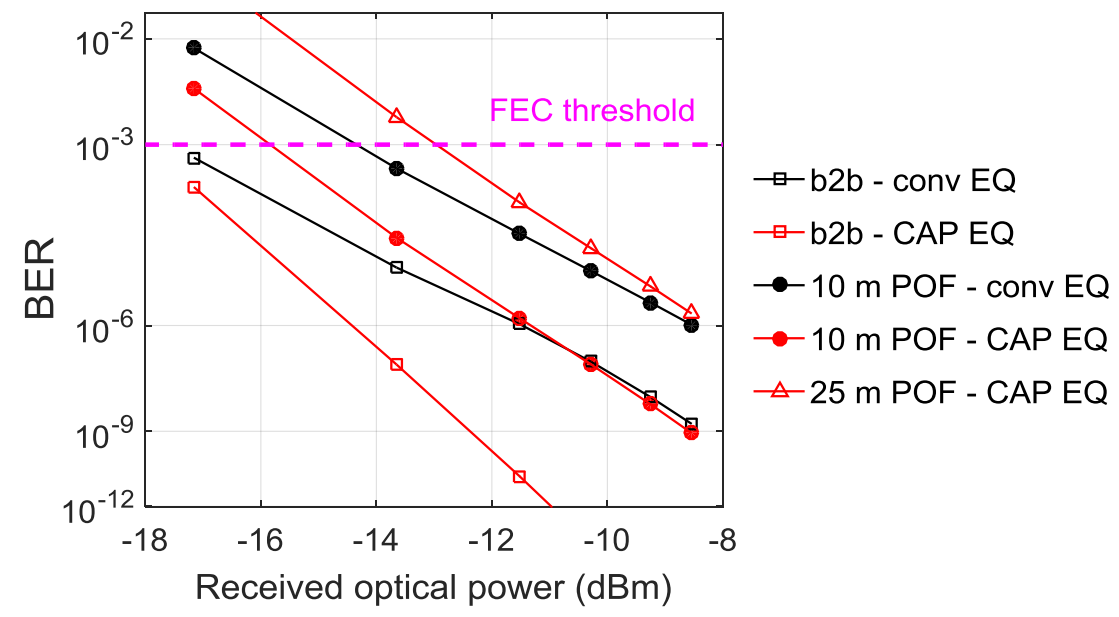

Figure 9. BER plots for the operating links for the different POF lengths and the two equalizers.

\section{CONCLUSIONS}

CAP modulation is an attractive spectral-efficient modulation scheme which is increasingly considered for use in free-space and fibre-based optical links to improve link capacity. However, the performance of CAP-based links can be severely impaired by ISI and CCI when the frequency response of the transmission channel is not ideal. Conventional equalization methods based on FFE and DFE equalizers fail to mitigate the induced CCI limiting therefore the achievable data rates and link reach. As a result, we have proposed the use of a new equalizer structure, named the CAP equalizer, for use in CAP-based transmission systems which can mitigate these impairments and significantly improve the performance of the links. Its operation relies on the use of the signal transmitted on both I- and Q- channels to recover the data transmitted on each of them. It can be implemented with minimal additional complexity over the conventional FFE and DFE equalizer. Its use is demonstrated in a $112 \mathrm{~Gb} / \mathrm{s}$ CAP-16 VCSEL-based OM4 MMF link and a $4 \mathrm{~Gb} / \mathrm{s}$ CAP-16 POF link. It is shown that the CAP equalizer enables longer reach for the two links ( $150 \mathrm{~m}$ and $25 \mathrm{~m}$ respectively) and improved receiver sensitivity over the conventional FFE and DFE equalizer with the same total number of taps. This new equalizer with its straightforward implementation paves the way to high-speed CAP-based short-reach optical links with improved performance.

\section{ACKNOWLEDGEMENTS}

The authors would like to acknowledge Keysight for their loan of the high bandwidth AWG (92 GS/s, M8196A), the University of Strathclyde for the provision of the micro-pixelated LED and the UK EPSRC for supporting this work via the UP-VLC (EP/K00042X/1) and TOWS (EP/S016570/1) projects. Data related to this work can be found in the University of Cambridge data repository https://doi.org/10.17863/CAM.51618.

\section{REFERENCES}

[1] Cisco, "Cisco Global Cloud Index: Forecast and Methodology, 2016-2021 White Paper," 2018.

[2] C. Kottke, C. Caspar, V. Jungnickel, R. Freund, M. Agustin, and N. N. Ledentsov, "High speed 160 Gb/s DMT VCSEL transmission using pre-equalization," in Optical Fiber Communications Conference (OFC), pp. 13, 2017.

[3] T. Zuo, L. Zhang, J. Zhou, Q. Zhang, E. Zhou, and G. N. Liu, "Single Lane 150-Gb/s, 100-Gb/s and 70-Gb/s 4-PAM Transmission over 100-m, 300-m and 500-m MMF Using 25-G Class 850nm VCSEL," in European Conference on Optical Communication (ECOC), pp. 974-976, 2016.

[4] R. Puerta, M. Agustin, L. Chorchos, J. Tonski, J. R. Kropp, N. Ledentsov, V. A. Shchukin, N. N. Ledentsov, R. Henker, I. T. Monroy, J. J. V. Olmos, and J. P. Turkiewicz, "Effective 100 Gb/s IM/DD 850-nm Multi- and Single-Mode VCSEL Transmission Through OM4 MMF," Journal of Lightwave Technology, vol. 35, pp. 423429, 2017.

[5] J. L. Wei, L. Geng, R. V. Penty, I. H. White, and D. G. Cunningham, "100 Gigabit Ethernet transmission enabled by carrierless amplitude and phase modulation using QAM receivers," in Optical Fiber Communication Conference (OFC), pp. 1-3, 2013.

[6] S. Vappangi and V. V. Mani, "Concurrent illumination and communication: A survey on Visible Light Communication," Physical Communication, vol. 33, pp. 90-114, 2019. 
[7] N. Bamiedakis, R. V. Penty, and I. H. White, "Carrierless amplitude and phase modulation in wireless visible light communication systems," Philosophical Transactions of the Royal Society A: Mathematical, Physical and Engineering Sciences, vol. 378, pp. 1-25, 2020.

[8] D. Karunatilaka, F. Zafar, V. Kalavally, and R. Parthiban, "LED Based Indoor Visible Light Communications: State of the Art," IEEE Communications Surveys \& Tutorials, vol. 17, pp. 1649-1678, 2015.

[9] S. Rajbhandari, J. McKendry, J. D., J. Herrnsdorf, H. Chun, G. Faulkner, H. Haas, I. M. Watson, D. O'Brien, and M. D. Dawson, "A review of gallium nitride LEDs for multi-gigabit-per-second visible light data communications," Semiconductor Science and Technology, vol. 32, p. 023001, 2017.

[10] I. N. Osahon, S. Rajbhandari, and W. O. Popoola, "SI-POF Transmission with CAP Modulation and SplitComplex MLP Equalizer," in IEEE International Conference on Communications (ICC), 2018, pp. 1-6.

[11] F. A. Sampaio, V. Silva, L. Neto, T. Ferreira, A. P. Barbero, and R. Ribeiro, "Highly Spectrally Efficient Discrete Multi-Tone Transmission over Step Index PMMA optical fibre for Short-Range Optical Communication," Journal of Communication and Information Systems, vol. 33, pp. 250-256, 2018.

[12] P. V. Mena, E. Ghillino, R. Dwigh, H. Satosh, M. Nakai, M. Kagami, and R. Scarmozzino, "Using system simulation to evaluate design choices for automotive ethernet over plastic optical fiber," in SPIE OPTO, pp. 1-9, 2018.

[13] J. Vinogradov, R. Kruglov, R. Engelbrecht, O. Ziemann, J. K. Sheu, K. L. Chi, J. M. Wun, and J. W. Shi, "GaN-Based Cyan Light-Emitting Diode with up to 1-GHz Bandwidth for High-Speed Transmission Over SIPOF," IEEE Photonics Journal, vol. 9, pp. 1-7, 2017.

[14] X. Li, N. Bamiedakis, W. Jinlong, J. J. D. McKendry, X. Enyuan, R. Ferreira, E. Gu, M. D. Dawson, R. V. Penty, and I. H. White, " $\mu$ LED-Based Single-Wavelength Bi-directional POF Link With $10 \mathrm{~Gb} / \mathrm{s}$ Aggregate Data Rate," Journal of Lightwave Technology, vol. 33, pp. 3571-3576, 2015.

[15] J. L. Wei, L. Geng, D. G. Cunningham, R. V. Penty, and I. H. White, "Gigabit NRZ, CAP and optical OFDM systems over POF links using LEDs," Opt. Express, vol. 20, pp. 22284-22289, 2012.

[16] J. J. Werner, "Tutorial on carrierless AM/PM," ANSI X3T9.5 TP/PMD Working Group, 1992-1993.

[17] X. Dong, N. Bamiedakis, D. G. Cunningham, R. V. Penty, and I. H. White, "A Novel Equalizer for 112 Gb/s CAP-Based Data Transmission Over 150 m MMF Links," Journal of Lightwave Technology, vol. 37, pp. 5937-5944, 2019.

[18] X. Dong, N. Bamiedakis, D. G. Cunningham, R. V. Penty, and I. H. White, "A Novel Equalizer for HighSpeed CAP-based Visible-Light Communication Links", submitted to IEEE Photonics Technology Letters, 2020. [19] M. Schuppert and C. A. Bunge, "5Gb/s Eye-Safe LED-Based SI-POF Transmission With Equalization of Transmitter Nonlinearities," IEEE Photonics Technology Letters, vol. 28, pp. 2732-2735, 2016.

[20] M. Schueppert, R. Kruglov, and C. A. Bunge, "Compensation of LED Nonlinearities in a Gigabit Polymer Optical Fiber Transmission link using a Combined Decision Feedback and Volterra-Series," in Photonic Networks; 16. ITG Symposium; Proceedings of, 2015, pp. 1-6.

[21] Y. Wang, L. Tao, X. Huang, J. Shi, and N. Chi, "Enhanced Performance of a High-Speed WDM CAP64 VLC System Employing Volterra Series-Based Nonlinear Equalizer," IEEE Photonics Journal, vol. 7, pp. 1-7, 2015.

[22] G. Stepniak, J. Siuzdak, and P. Zwierko, "Compensation of a VLC Phosphorescent White LED Nonlinearity by Means of Volterra DFE," IEEE Photonics Technology Letters, vol. 25, pp. 1597-1600, 2013.

[23] X. Lu, K. Wang, L. Qiao, W. Zhou, Y. Wang, and N. Chi, "Nonlinear Compensation of Multi-CAP VLC System Employing Clustering Algorithm Based Perception Decision," IEEE Photonics Journal, vol. 9, pp. 1-9, 2017.

[24] X. Lu, L. Qiao, Y. Zhou, W. Yu, and N. Chi, "An I-Q-Time 3-dimensional post-equalization algorithm based on DBSCAN of machine learning in CAP VLC system," Optics Communications, vol. 430, pp. 299-303, 2019.

[25] X. Lu, M. Zhao, L. Qiao, and N. Chi, "Non-linear Compensation of Multi-CAP VLC System Employing Pre-Distortion Base on Clustering of Machine Learning," in Optical Fiber Communication Conference (OFC), San Diego, California, 2018, p. paper M2K.1.

[26] R. X. G. Ferreira, E. Xie, J. J. D. McKendry, S. Rajbhandari, H. Chun, G. Faulkner, S. Watson, A. E. Kelly, E. Gu, R. V. Penty, I. H. White, D. C. O'Brien, and M. D. Dawson, "High Bandwidth GaN-Based Micro-LEDs for Multi-Gb/s Visible Light Communications," IEEE Photonics Technology Letters, vol. 28, pp. 2023-2026, 2016. 